Original Article: Cystic Fibrosis

\title{
Prevalence of Hearing and Vestibular Loss in Cystic Fibrosis Patients Exposed to Aminoglycosides. ${ }^{1}$
}

\author{
Jaynee A. Handelsman, Ph.D. ${ }^{1,2}$,
}

Samya Z. Nasr 0000-0002-4124-316X 0000-0002-4124-316X, M.D., C.P.I. ${ }^{3}$, Crystal Pitts, Au.D. ${ }^{2}$,

W. Michael King, Ph.D. ${ }^{1}$

${ }^{1}$ University of Michigan Medical School, Ann Arbor, Michigan

${ }^{2}$ University of Michigan Health System, Ann Arbor, Michigan

${ }^{3}$ Division of Pediatric Pulmonology, Department of Pediatrics and Communicable Diseases, University of Michigan Health System, Ann Arbor,

Address correspondence to: Jaynee Handelsman, Ph.D., Pediatric Otolaryngology Head and Neck Surgery, 1540 E. Hospital Dr., SPC 4241, Ann Arbor, MI 48109-4241, 734-936-9415 (voice)/734-763-7802 (fax), jaynee@ med.umich.edu.

Hearing and Vestibular loss in aminoglycosides treated CF patients

This work was partially supported by the Cystic Fibrosis Foundation, grant number N008681385013.

Key Words: ototoxicity; aminoglycosides; vestibular loss; hearing loss; cystic fibrosis

Abbreviations: CF: Cystic Fibrosis

\footnotetext{
${ }^{1}$ This is the author manuscript accepted for publication and has undergone full peer review but has not been through the copyediting, typesetting, pagination and proofreading process, which may lead to differences between this version and the Version of Record. Please cite this article as doi:10.1002/ppul.23763
} 
AGS: aminoglycosides

VTC: Vestibular Testing Center

DVA: Dynamic visual acuity

VNG: Testing to determine the presence of oscillopsia, videonystagmography

SCEV: Peak slow component eye velocity (SCEV)

VOR: Vestibulo-ocular reflex

DPOAE: Distortion Product Otoacoustic Emissions

vHIT: the video head impulse test

VEMP: vestibular evoked myogenic potential

\section{Summary/ Abstract}

Aim: Cystic Fibrosis (CF) patients frequently use aminoglycosides (AGS) to treat CF exacerbation due to colonization with Pseudomonas aeruginosa. Although AGS can cause vestibular and auditory sensory losses that can negatively impact quality of life, little is known about the prevalence of vestibular loss in this population. The aim of this study was to determine the prevalence of hearing loss and/or vestibular dysfunction in CF patients treated with AGS. Methods: The relationship between hearing status and vestibular status was also investigated. Hearing was determined to be normal or abnormal based on pure tone air and bone conduction thresholds. Vestibular outcome was divided into four categories; normal, non-lateralized vestibular dysfunction, unilateral loss, and bilateral loss based on results of post head shaking testing, positional and positioning testing, bithermal calorics, sinusoidal and rotational step 
testing. Results: Of our cohort of 71 patients, 56 (79\%) patients have vestibular system dysfunction while only 15 (21\%) have normal vestibular system function. Overall, 16 patients $(23 \%)$ have hearing loss. In considering the relationship between auditory and vestibular function, $12(17 \%)$ demonstrated both normal hearing and normal vestibular function and 13 (18\%) have both hearing loss and abnormal vestibular function. Of the 55 (78\%) patients with normal hearing, $43(61 \%)$ have vestibular dysfunction, while $3(4 \%)$ of patients with normal vestibular function have hearing loss. Conclusion: These results suggest that monitoring hearing alone is insufficient to detect ototoxicity in CF patients being treated with systemic AGS.

\section{Introduction}

Cystic Fibrosis $(\mathrm{CF})$ is the most common life threatening, autosomal recessive disorder in the Caucasian population ${ }^{1}$. Patients with CF constitute a group that use aminoglycosides (AGS) at a high rate due to the chronic colonization by Pseudomonas aeruginosa (P. aeruginosa) and frequent pulmonary exacerbation ${ }^{2-5}$. Aminoglycoside antibiotics can cause vestibular and auditory sensory losses that can seriously impact a patient's quality of life ${ }^{6-7}$. Loss of auditory function has been well documented by otoacoustic emission testing and clinical audiometry ${ }^{8-14}$. However, the prevalence of vestibular dysfunction/toxicity among patients exposed to AGS has not been reported. We could find only two systematic studies of vestibular disorders in $\mathrm{CF}$ patients. Both studies used caloric irrigation asymmetry to assess function and both were restricted to CF patients who received tobramycin. Thomsen et al. ${ }^{15}$ evaluated 53 pediatric patients, only one of which had a caloric asymmetry. Scheenstra et al. ${ }^{16}$ evaluated 23 adult CF patients who received systemic tobramycin and reported the prevalence of vestibular disorders as $30.4 \%$ of their population. Complicating factors in identifying the prevalence of vestibular 
dysfunction are the variable presentation of symptoms within individual patients, the variable compensatory adjustments of the symptoms, and the difficulty in measuring vestibular function in pediatric patients. Furthermore, there are no commonly accepted protocols for monitoring vestibular function, especially in children ${ }^{17-20}$. In addition, CF patients typically receive multiple AGS treatments that may include gentamicin and amikacin as well as tobramycin (systemically and inhaled). These factors lead to difficulty is estimating the prevalence of vestibular dysfunction in this patient population ${ }^{21-24}$.

There is an urgent need to assess the prevalence of ototoxicity in CF patients. First, as noted on the Cystic Fibrosis Foundation Patient Registry, more than 75 percent of people with CF are diagnosed by age 2 and there is a steady increase in the median survival age of CF patients to over 40 years of age; more than half of these patients are older than 18 years of age ${ }^{25-}$ 30. Second, multiple exposures to AGS in CF patients can lead to cumulative damage to the auditory and vestibular systems over the lifetime of the patients, the symptoms of which are largely irreversible and disabling.

Symptoms of vestibular dysfunction may include oscillopsia (disturbed vision with head motion), dizziness, motion sickness, and unsteadiness when standing or walking, especially in the dark ${ }^{31-36}$. These symptoms negatively impact patients' quality of life and can lead to significant disability including an increased rate of falling. Despite the serious impact of ototoxicity and vestibular dysfunction on quality of life, the two studies cited above are the only systematic studies of vestibular ototoxicity in patients ${ }^{15-16}$. In fact, much of the literature on aminoglycoside ototoxicity is devoted to kidney disease and/or auditory dysfunction ${ }^{37-38}$. For this reason, we conducted a study to establish the prevalence of vestibular system involvement in patients treated with systemic AGS for treatment of CF pulmonary exacerbation. Hearing was This article is protected by copyright. All rights reserved. 
also evaluated to determine the relationship between auditory and vestibular loss in individual patients.

\section{Methods}

\section{Patient Selection:}

CF Patients, 8 years of age and older who attended the University of Michigan CF Center and received intravenous AGS for treatment of pulmonary exacerbation or pneumonia, were eligible to participate in this study. Patients less than 8 years were excluded from the study because of concerns that they would not be able to fully cooperate with the testing as well as concerns about appropriate diagnostic criteria in young children ${ }^{39,19}$. Testing was conducted as close to the start of a treatment course as possible although we did not control for the number of prior exposures to AGS. As a result, some patients had multiple courses of AGS prior to testing. While most of the patients' exposures to intravenous AGS were limited to tobramycin and/or gentamicin, some patients were treated with intravenous amikacin. Finally, because this patient population is frequently treated with multiple antibiotics over time, both inhaled and intravenous, the aminoglycoside exposure was variable within and across patients.

Auditory and vestibular testing was completed in the Vestibular Testing Center (VTC) at the University of Michigan Health System. Prior to audiologic or vestibular testing, otoscopic inspection was performed in order to determine that the patients' ear canals were free of cerumen or other debris that would obstruct visualization of the tympanic membrane and might affect the auditory thresholds or interfere with caloric irrigation during vestibular testing.

\section{Audiologic Assessment:}

This article is protected by copyright. All rights reserved. 
Audiologic testing for subjects in this study was performed at the time of enrollment or at the time of vestibular testing. Testing consisted of pure tone air conduction behavioral thresholds at octave frequencies from $250-8,000 \mathrm{~Hz}$. When evidence of hearing loss was present, bone conduction testing was conducted at octave frequencies from $250-4000 \mathrm{~Hz}$. Acoustic immittance testing (tympanometry and acoustic reflexes) was completed to rule out the presence of middle ear pathology.

Vestibular Assessment:

A case history was obtained to ascertain whether the subjects had prior symptoms related to vestibular dysfunction or other co-morbidities that might impact vestibular function. The vestibular evaluation included completion of dynamic visual acuity (DVA) testing to determine the presence of oscillopsia, videonystagmography (VNG), and sinusoidal and rotational step testing. The procedures for each portion of the battery are summarized in the following paragraphs.

DVA was assessed by asking subjects to read from a Snellen eye chart positioned at a distance of $10 \mathrm{ft}$. Visual acuity was determined while the patient's head was still and with head rotation back and forth at 1-2 Hz. A change in visual acuity with head rotation of more than two lines was considered to be abnormal and suggestive of oscillopsia.

VNG testing included recording horizontal and vertical eye movements for each eye using infrared cameras and digital video technology. All of the video data were digitally stored for analysis. Since eye movements form the basis of our vestibular assessment, tests of visual fixation, smooth pursuit, and random saccades were performed to rule out oculomotor disorders. The remaining tests were completed with vision denied to prevent visual suppression of the 
vestibular response. Following oculomotor testing, eye movements were recorded with the patient's head and body in various positions as well as following rapid head shaking. Eye movements were evaluated for the presence of nystagmus in any condition. Alternate bithermal caloric irrigations using warm and cool water (open loop system) were performed to evaluate the response of the horizontal semicircular canal of each ear independently. Eye movements were recorded post irrigation and examined for the presence of nystagmus. Peak slow component eye velocity (SCEV) during nystagmus is the relevant dependent variable that is computed from the video data. The total SCEV for each ear was compared to determine whether a significant asymmetry in the vestibulo-ocular reflex (VOR) was present based on the clinical criteria used in the VTC. Specifically, a significant asymmetry was defined as a unilateral weakness $>25 \%$.

Sinusoidal rotary chair testing was conducted over a range of frequencies from 0.01 to $1.28 \mathrm{~Hz}$. Unlike caloric tests, which employ non-physiological stimuli, the rotary chair test employs a natural stimulus to excite the vestibular system bilaterally. For each stimulus frequency, the phase, gain, and asymmetry of the patient's eye movements were calculated. Gain is defined as the ratio of the eye movements (SCEV) compared to the speed of the chair movement, phase is a measure of the timing relationship between the eyes and the chair, and asymmetry is the relative gain for clockwise and counter clockwise rotations.

Patients with bilateral vestibular loss usually present with decreased gain values, especially at the lowest test frequencies. For frequencies at which there are adequate gain responses to determine phase and symmetry values (>0.2), increased phase leads may also be present. With unilateral vestibular loss, increased phase leads (with or without asymmetries) are often present. 
Rotational step testing was also performed during which each patient was abruptly accelerated to a high angular velocity $(100 \mathrm{deg} / \mathrm{sec}$ or $240 \mathrm{deg} / \mathrm{sec})$, which typically resulted in a brisk nystagmus. Rotation continued at a constant speed long enough for the nystagmus to subside. After a period of constant velocity rotation, the patient was abruptly decelerated to a stop, which was experienced as acceleration in the opposite direction and was accompanied by another burst of brisk nystagmus that decayed over time. Step testing included both clockwise and counterclockwise acceleration and the deceleration from each. The dependent variables of interest are the peak SCEV for each of the four steps as well as the decay time, which is referred to as the VOR time constant. Bilateral vestibular loss results in gain reductions and short time constants. Short time constants may also be present in unilateral and non-lateralizing vestibular loss.

For each portion of the test battery individual subject's data were determined to be normal or abnormal based on the clinical criteria that are used for all patients who are evaluated in the VTC. These criteria are derived from norms that were established by equipment manufacturers and our own clinical data. Typically, a result is considered clinically significant if it exceeds two standard deviations from the mean values for normal subjects.

The study was approved by The University of Michigan Institutional Review Board (IRB).

\section{Results}

Vestibular test results for each patient were described as falling into one of four categories. Specifically, they were considered to be normal if the findings on each of the portions 
of the test battery fell within the normal range based upon our clinical criteria. When that was not the case, they fell into one of three abnormal categories as seen in Table 1. Category A includes non-lateralizing evidence of peripheral vestibular system involvement, such as the presence of spontaneous, post head shaking, or positional nystagmus, increased phase leads and/or asymmetries in sinusoidal rotational testing, or evidence of a short time constant in rotational step testing. To be included in this category, a patient's data demonstrated one or more abnormal findings as mentioned above. Category B represents evidence of a unilateral peripheral vestibular loss based upon a significant asymmetry in caloric results. Findings that are included in Category A may also be present. Category $\mathrm{C}$ represents evidence of a bilateral peripheral vestibular loss including bilateral reductions in caloric findings and/or gain reductions in rotational testing at one or more frequencies. One or more findings that are included in Category A, as well as evidence of an asymmetry, may also be present.

The hearing status of individual patients was separated into two categories: normal or hearing loss. Patients were considered to have normal hearing when behavioral thresholds at all standard audiometric frequencies fell within the normal range (i.e. $<25 \mathrm{~dB} \mathrm{HL}$ ) for air conduction and bone conduction. Although Distortion Product Otoacoustic Emissions (DPOAE) were recorded for some patients, the data presented for this cohort is limited to tests that were completed for every participant. High frequency pure tone thresholds were not obtained due to equipment limitations.

The patients' vestibular data to date are summarized in Figure 1. We have completed vestibular testing on 71 patients, ranging in age from 8-56 years $($ mean $=18.3$ years, median $=$ 17 years). The sample included 29 (41\%) males and $42(59 \%)$ females. Of the 71 patients, 27 (38\%) have evidence of non-lateralized peripheral vestibular system involvement, $8(11 \%)$ have This article is protected by copyright. All rights reserved. 
significant unilateral vestibular losses, and $21(30 \%)$ have evidence of bilateral vestibular system paresis. Of the sample population, 56 (79\%) individuals demonstrated the presence of vestibular system dysfunction while only 15 individuals (21\%) have no evidence of vestibular system involvement based upon the results of testing. In terms of hearing status, 16 patients (23\%) have evidence of hearing loss. Of note, the prevalence of vestibular loss in this patient population is much greater than is the prevalence of hearing loss. Additionally, a cursory review of patients' AGS exposures revealed no apparent relationship between specific antibiotic exposure at the time of testing and hearing loss or vestibular loss. For example, normal hearing and hearing loss occurred for all three AGS as did normal vestibular function and vestibular loss. Although not a primary objective of this study, our data do not provide evidence that specific medications are primarily cochleotoxic or vestibulotoxic.

We were also interested in examining the relationship between vestibular loss and hearing status, the results of which is displayed in Table 2. Of the patient population evaluated, only $12(17 \%)$ demonstrated both normal hearing and normal vestibular function and $13(18 \%)$ showed evidence of both hearing loss and abnormal vestibular function. Of the 55 (78\%) patients with normal hearing, 43 (61\%) have vestibular dysfunction based upon the results of testing, and $3(4 \%)$ of patients with normal vestibular function have hearing loss. Based on these data, there is no clear relationship between hearing status and vestibular function in this group.

\section{Discussion}

These data demonstrate that our overall prevalence rate of vestibular dysfunction of $79 \%$ (38\% with non-lateralizing peripheral involvement and $41 \%$ with either a significant unilateral or bilateral paresis) in our cohort of CF patients who were exposed to systemic aminoglycosides is 
much higher than previously recognized ${ }^{32}$. Scheenstra et al. included subjects exposed to one or more courses of systemic tobramycin and who had not received any other ototoxic aminoglycoside. In contrast, some of the patients included in our study were exposed to multiple AGS including gentamicin, tobramycin, and/or amikacin. Because of incomplete medical records, we were unable to accurately determine the cumulative dosages of aminoglycoside antibiotics received by our patients. Nevertheless, the prevalence of vestibular ototoxicity in our sample of CF patients is an important finding that argues for more systematic testing of vestibular function in this patient population. Despite the awareness and attention given to the risk of hearing loss in patients treated with AGS, our data demonstrate that there is an even greater risk of vestibular dysfunction and its attendant disabling symptoms.

Vestibular testing of CF patients is infrequently performed at most CF centers. The most likely explanations for lack of testing are insufficient knowledge of the symptoms and prevalence of vestibular disorders and the complexity of the tests themselves. For example, although "dizziness" is often cited as a vestibular symptom, it is not as common as oscillopsia or decreased visual acuity among patients with bilateral vestibular dysfunction. Additionally, patients may underreport symptoms. For example, among our patients, although most denied symptoms of oscillopsia when questioned, all of the patients with bilateral vestibular loss demonstrated impaired dynamic visual acuity when tested. Furthermore, patients may compensate for vestibular losses and be asymptomatic after an ototoxic insult; however, cell losses in the peripheral labyrinth caused by ototoxicity are cumulative (sensory cells do not regenerate) $7,17,20,31,39$. Subsequent ototoxic insults will eventually exhaust the brain's compensatory capacity. 
The complexity of vestibular testing and discomfort to the patient are also barriers to systematic evaluation. Only a small number of our young pediatric patients were willing to submit to a complete vestibular test battery and an even smaller number were willing to be retested after subsequent exposures to AGS. These patients were particularly reluctant to receive caloric and rotary chair tests, which represent the gold standards for vestibular evaluation. The development of new vestibular tests such as the video head impulse test (vHIT) and vestibular evoked myogenic potential (VEMP) tests may be better tolerated by CF patients as can be performed in less time with less physical or psychological discomfort. Additionally, creation of facilities and protocols that are adapted for children may be helpful. However, systematic studies to determine the sensitivity and specificity of these tests relative to rotary chair and caloric tests in adult and pediatric patient populations are still needed.

Our data also demonstrate that the prevalence of hearing loss in our cohort of patients was relatively low at $22 \%$. In addition, there is no discernable relationship between hearing status and vestibular function in CF patients exposed to aminoglycoside therapies. Specifically, $61 \%$ of patients with vestibular loss have normal hearing and $4 \%$ of patients with normal vestibular function have hearing loss. Furthermore, there are patients with normal hearing represented in all three categories of vestibular disorders. An important limitation of our study related to its sensitivity in identifying hearing loss is that data are limited to standard frequencies. In a recent investigation, Al-Malky and colleagues ${ }^{40}$ reported a similar prevalence rate of $21 \%$ using standard audiometry whereas the prevalence increased to $24 \%$ using extended high frequency (EHF) audiometry. Additionally, DPOAE results were significantly correlated with EHF results in their cohort. Importantly, these results suggest that monitoring auditory function alone in this population of patients is an insufficient way to look for evidence of ototoxicity in 
individual patients with CF being treated with systemic AGS. While it is apparent that high frequency audiometry and DPOAE testing may be more sensitive measures of auditory sensory loss in this patient population, the prevalence of vestibular loss in our patient population is much greater than hearing loss. Furthermore, because of the substantial negative impact of vestibular loss on function and quality of life, early identification is critical.

Providing patients and families with information about the role of the vestibular system is important, particular related to maintenance of clear vision with head movements and in postural control and balance. When treatment with systemic AGS is necessary, having an understanding of the potential effects of ototoxicity and its associated symptoms will enable patients to identify early changes in order to alert their health care team. In addition, early identification of vestibular loss provides an opportunity to counsel patients and families about important safety issues. Patients in our cohort were counseled about the test results and their implications by the audiologist who performed testing. Based on the results of this investigation, obtaining baseline measures of auditory and vestibular function as well as monitoring function in conjunction with each aminoglycoside exposures are warranted. Early diagnosis of sensory loss may enable health care providers to alter treatment in some cases. When that is not possible, knowing about vestibular loss can facilitate timely referral to vestibular physical therapy when rehabilitation is indicated to improve gaze stabilization, promote sensory substitution in the case of bilateral vestibular loss, and facilitate habituation of symptoms. Our patients were offered appointments for physical therapy when appropriate.

As has been discussed, we did not control for the number or type of aminoglycoside exposure for this study. We also did not investigate whether serial changes in function with repeated exposure are detectable. As a result, additional studies that attempt to look at vestibular This article is protected by copyright. All rights reserved. 
function relative to the type and dosage of aminoglycosides administered (both inhaled and intravenous) and the cumulative impact of repeated exposures are indicated to further define these relationships.

Acknowledgements. This study was partially supported by a grant from the Cystic Fibrosis Foundation, grant number N008681-385013.

\section{References}

1. Saiman LM, Anstead M, Mayer-Hamblett N. Effect of azithromycin on pulmonary function in patients with cystic fibrosis uninfected with pseudomonas aeruginosa: a randomized controlled trial. JAMA 2010;303(17):1707-1715.

2. Martins LMN, Camargos PAM, Becker HMG, Becker CG, Guimaraes RES. Hearing loss in cystic fibrosis. International Journal of Pediatric Otorhinolaryngology 2010; 74:469-473.

3. Banerjee D, Stableforth $\mathrm{D}$. The treatment of respiratory pseudomonas infection in cystic fibrosis: what drug and which way? Drugs 2000; 60:1053-64.

4. Pressler T, Bohmova C, Conway S, Dumcius S, Hjelte L, Høiby N, Kollberg H, Tümmler B, Vavrova V. Chronic pseudomonas aeruginosa infection definition: EuroCareCF working group report. J Cyst Fibros 2011;10(Suppl 2): S75-8.

5. Smyth A. Update on treatment of pulmonary exacerbations in cystic fibrosis. Curr Opin Pulm Med 2006; 12:440-4.

6. Walsh RM, Bath AP, Bance ML. Reversible tobramycin-induced bilateral high-frequency vestibular toxicity. ORL J Otorhinolaryngol Relat Spec 2000;62(3):156-159. 
7. Calder JH, Jacobson GP. Acquired bilateral peripheral vestibular system impairment: rehabilitative options and potential outcomes. J Am Acad Audiol, 2000;11:514-521.

8. Al-Malky G, Suri R, Dawson SJ, Sirimanna T, Kemp D. Aminoglycoside antibiotics cochleotoxicity in paediatric cystic fibrosis (CF) patients: a study using extended high-frequency audiometry and distortion product otoacoustic emissions. Int J Audiol 2011; 50:112-22.

9. Hennig S, McKay K, Vidmar S, O'Brien K, Stacey S, Cheney J, Wainwright CE. Safety of inhaled (Tobi®) and intravenous tobramycin in young children with cystic fibrosis. J Cyst Fibros $2014 ; 13: 428-34$.

10. Nakashima T, Teranishi M, Hibi T, Kobayashi M, Umemura M. Vestibular and cochlear toxicity of aminoglycosides-a review. Acta Oto-Laryngologica 2000; 120:904-11.

11. Prayle A, Watson A, Fortnum H, Smyth A. Side effects of aminoglycosides on the kidney, ear and balance in cystic fibrosis. Thorax 2010; 65:654-8.

12. Scott CS, Retsch-Bogart GZ, Henry MM. Renal failure and vestibular toxicity in an adolescent with cystic fibrosis receiving gentamicin and standard-dose ibuprofen. Pediatr Pulmonol 2001; 31:314-6.

13. Leigh-Paffenroth E. Objective measures of ototoxicity. Hearing and Hearing Disorders: Research and Diagnostics. 2005;9(1):10-16.

14. Konrad-Martin D, Gordon JS, Reavis KM, Wilmington DJ, Helt WJ, Fausti SA. Audiological monitoring of patients receiving ototoxic drugs. Hearing and hearing Disorders: Research and Diagnostics. 2005;9(1)17-21. 
15. Thomsen J, Friis B, Jensen K, Bak-Pedersen K, Larsen PK. Tobramycin ototoxicity. Repeated courses of high dosage treatment in children with cystic fibrosis. J Antimicrob Chemother. 1979; 5:257-60.

16. Scheenstra RJ, Rijntjes E, Tavy DL, Kingma H, Heijerman HG. Vestibulotoxicity as a consequence of systemically administered tobramycin in cystic fibrosis patients. Acta otolaryngologica. 2009; 129:4-7.

17. Paydarfar JA, Goebel JA. Integrated clinical and laboratory vestibular evaluation. Current Opinion in Otolaryngology \& Head and Neck Surgery. 2000; 8:363-8.

18. Taylor J, Goodkin HP. Dizziness and vertigo in the adolescent. Otolaryngol Clin North Am. 2011; 44:309-21, vii-viii.

19. Valente LM. Assessment techniques for vestibular evaluation in pediatric patients. Otolaryngol Clin North Am. 2011; 44:273-90, vii.

20. Wiener-Vacher S. What is useful in vestibular testing? Oto-Rhino-Laryngologica. 2002; 11:95-8.

21. Wiener-Vacher SR. Vestibular disorders in children. Int J Audiol. 2008; 47:578-83

22. Mulheran MC, Degg S, Burr DW, Morgan DE, Stableforth. Occurrence and risk of cochleotoxicity in cystic fibrosis patients receiving and repeated high doses of aminoglycoside therapy. Antimicrobial Agents and Chemotherapy. 2001;45(9):2502-2509.

23. Tan HVK. Mulheran M, Knox AJ, Smyth AR. Aminoglycoside prescribing and surveillance in cystic fibrosis. American Journal of Critical Care in Medicine. 2003; 167:819-823. 
24. Handelsman JA. Audiologic findings in vestibular toxicity. Pharmacology and ototoxicity for Audiologists. 2006;272-286.

25. Besier T, Goldbeck L. Growing up with cystic fibrosis: achievement, life satisfaction, and mental health. Qual Life Res. 2012; 21:1829-35.

26. Chamnan P, Shine BS, Haworth CS, Bilton D, Adler AI. Diabetes as a determinant of mortality in cystic fibrosis. Diabetes Care. 2010; 33:311-6.

27. Cohen-Cymberknoh M, Shoseyov D, Kerem E. Managing cystic fibrosis: strategies that increase life expectancy and improve quality of life. Am J Respir Crit Care Med. 2011; 183:1463-71.

28. Nick JA, Chacon CS, Brayshaw SJ, Jones MC, Barboa CM, St Clair CG, Young RL, Nichols DP, Janssen JS, Huitt GA, Iseman MD, et al. Effects of gender and age at diagnosis on disease progression in long-term survivors of cystic fibrosis. Am J Respir Crit Care Med. 2010; 182:61426.

29. Simmonds NJ, D'Souza L, Roughton M, Alton EW, Davies JC, Hodson ME. Cystic fibrosis and survival to 40 years: a study of cystic fibrosis transmembrane conductance regulator function. Eur Respir J. 2011; 37:1076-82.

30. Cystic Fibrosis Foundation [US]. https://www.cff.org/What-is-CF/About-Cystic-Fibrosis/. Accessed 12.17.2016.

31. Ahmed RM, Hannigan IP, MacDougall HG, Chan RC, Halmagyi GM. Gentamicin ototoxicity: a 23-year selected case series of 103 patients. Med J Aust. 2012; 196:701-4. 
32. Black FO, Gianna-Poulin C, Pesznecker SC. Recovery from vestibular ototoxicity. Otol Neurotol. 2001; 22:662-71.

33. Black FO, Pesznecker S, Stallings V. Permanent gentamicin vestibulotoxicity. Otol Neurotol. 2004; 25:559-69.

34. Ishiyama G, Ishiyama A, Kerber K, Baloh RW. Gentamicin ototoxicity: clinical features and the effect on the human vestibulo-ocular reflex. Acta Otolaryngol. 2006; 126:1057-61.

35. Handelsman JA. Vestibular ototoxicity. Hearing and hearing Disorders: Research and Diagnostics. 2005;9(1): 26-30.

36. Brandt T. Bilateral vestibulopathy revisited. Eur J Med Res. 1996;1(8):361-368.

37. Prayle A, Watson A, Fortnum H, Smyth A. Side effects of aminoglycosides on the kidney, ear and balance in cystic fibrosis. Thorax. 2010; 65:654-8.

38. Prayle A, Smyth AR. Aminoglycoside use in cystic fibrosis: therapeutic strategies and toxicity. Curr Opin Pulm Med. 2010; 16:604-10.

39. O'Reilly R, Grindle C, Zwicky EF, Morlet T. Development of the vestibular system and balance function: differential diagnosis in the pediatric population. Otolaryngol Clin North Am. 2011; 44:251-71, vii.

40. Al-Malky G, Dawson SJ, Sirimanna T, Bagkeris E, Suri R. High-frequency audiometry reveals high prevalence of aminoglycoside ototoxicity in children with cystic fibrosis. J Cyst Fibros. 2011; 14: 248-54. 
Figure 1. Prevalence of vestibular and auditory dysfunction in CF patients who received one or more intravenous courses of aminoglycoside antibiotics.

Table 1. Criteria for each category of vestibular disorders.

\begin{tabular}{|l|l|l|}
\hline $\begin{array}{l}\text { Nonlateralized Peripheral } \\
\text { A) }\end{array}$ & $\begin{array}{l}\text { Unilateral Vestibular Paresis } \\
\text { (Category B) }\end{array}$ & $\begin{array}{l}\text { Bilateral Vestibular Paresis } \\
\text { (Category C) }\end{array}$ \\
\hline $\begin{array}{l}\text { Spontaneous nystagmus } \\
\text { nystagmus }\end{array}$ & $\begin{array}{l}\text { Caloric inter-ear asymmetry } \\
\text { greater than or equal to 25\% }\end{array}$ & $\begin{array}{l}\text { Each of the four caloric } \\
\text { responses less than } 10 \mathrm{deg} / \mathrm{sec}\end{array}$ \\
$\begin{array}{l}\text { Significant positional } \\
\text { nystagmus }\end{array}$ & $\begin{array}{l}\text { One or more of the results } \\
\text { listed in Category A may also } \\
\text { be present }\end{array}$ & $\begin{array}{l}\text { Gain reductions in rotational } \\
\text { step testing }\end{array}$ \\
$\begin{array}{l}\text { Short time constants for } \\
\text { rotational steps }\end{array}$ & $\begin{array}{l}\text { Gain reductions in sinusoidal } \\
\text { rotational testing }\end{array}$ \\
$\begin{array}{l}\text { Increased phase leads at any } \\
\text { sinusoidal frequency }\end{array}$ & $\begin{array}{l}\text { One or more of the results } \\
\text { listed in Category A may also } \\
\text { be present }\end{array}$ \\
\begin{tabular}{l} 
Significant chair asymmetries \\
\hline
\end{tabular}
\end{tabular}

Table 2. Co-morbidity of vestibular and auditory losses in CF patients.

\begin{tabular}{|l|l|l|}
\hline & Normal Auditory & Abnormal Auditory \\
\hline Normal Vestibular & 12 & 3 \\
\hline Abnormal Vestibular & 43 & 13 \\
\hline
\end{tabular}


Auditory and Vestibular Function in Total Sample of CF Patients Tested $(n=71)$

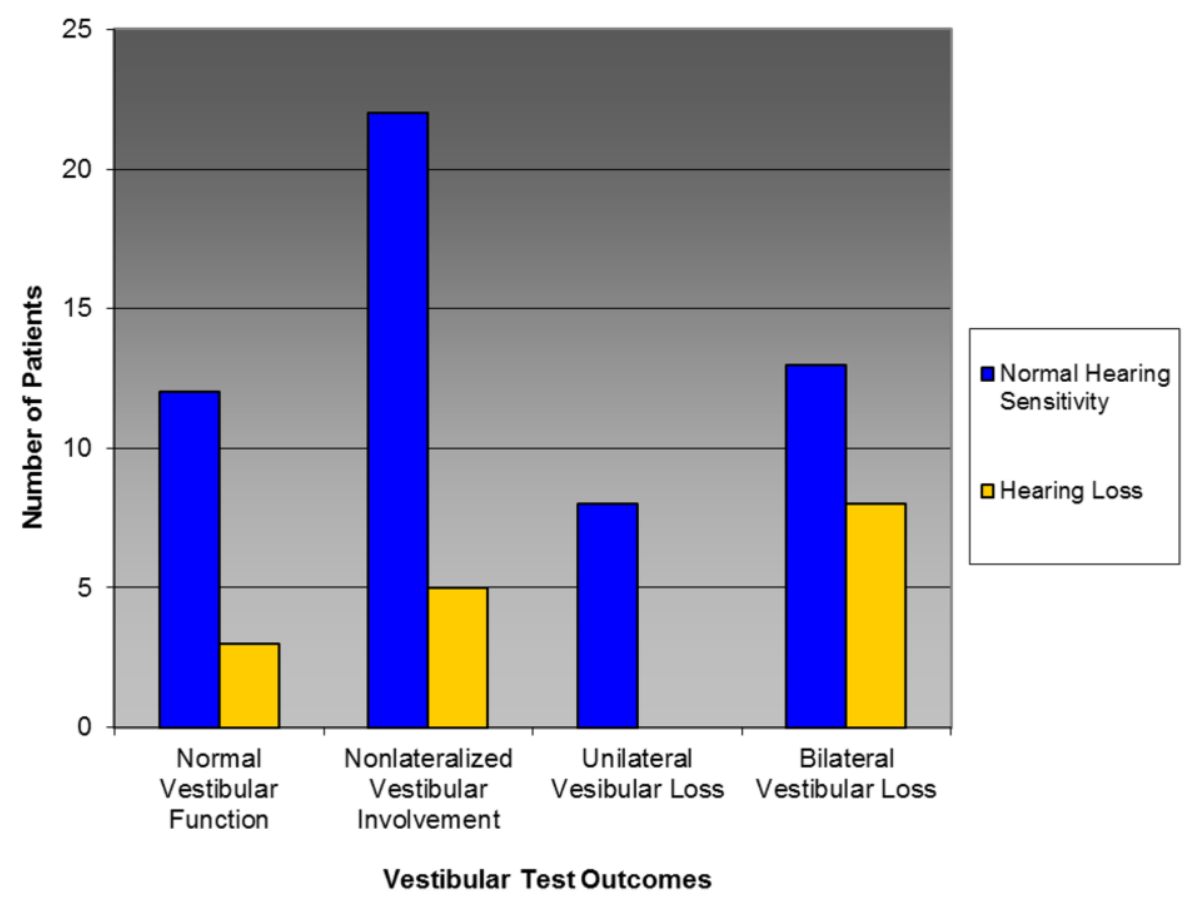

Figure 1

This article is protected by copyright. All rights reserved. 